Research Paper

\title{
Difference in the short-term outcomes of laparoscopic gastrectomy for gastric carcinoma between the east and west: a retrospective study from the IMIGASTRIC trial
}

\author{
Jian-Xian Lin'1,2\#, Jun-Peng Lin²,2\#, Jacopo Desiderio3\#, Jian-Wei Xie1,2, Alessandro Gemini3 ${ }^{3}$, Jia-bin Wang1,2,

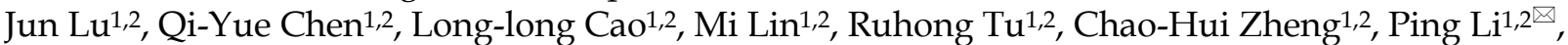 \\ Amilcare Parisi ${ }^{\circledR}$, Chang-Ming Huang ${ }^{1,2}{ }^{\bowtie}$ \\ 1. Department of Gastric Surgery, Fujian Medical University Union Hospital, Fuzhou, Fujian Province, China \\ 2. Key Laboratory of Ministry of Education of Gastrointestinal Cancer, Fujian Medical University, Fuzhou, Fujian Province, China \\ 3. Department of Digestive Surgery, St. Mary's Hospital, University of Perugia, Terni, Italy \\ \#Lin JX, Lin JP, and Desiderio J contributed equally to this work and should be considered co-first authors.
}

$\square$ Corresponding authors: Amilcare Parisi, E-mail: amilcareparisi@virgilio.it; Department of Digestive Surgery, St. Mary's Hospital, University of Perugia, Terni, Italy. Ping Li, E-mail: 24627878@qq.com; Chang-Ming Huang, E-mail: hcmlr2002@163.com; Department of Gastric Surgery, Fujian Medical University Union Hospital, No.29 Xinquan Road, Fuzhou 350001, Fujian Province, China.

(c) The author(s). This is an open access article distributed under the terms of the Creative Commons Attribution License (https://creativecommons.org/licenses/by/4.0/). See http://ivyspring.com/terms for full terms and conditions.

Received: 2018.11.03; Accepted: 2019.04.23; Published: 2019.07.10

\begin{abstract}
Purpose: To compare the clinicopathologic data and short-term surgical outcomes of laparoscopic gastrectomy (LG) for gastric cancer (GC) between the east and west.

Methods: Patient demographics, surgical procedures, pathological information, and postoperative recovery were compared among gastric cancer patients who underwent LG in the clinical trial of IMIGASTRIC (NCT02325453) between 2009 and 2016.

Results: More younger males, higher BMI, lower ASA score and less neoadjvant chemotherapy were evident in east patient cohort. Eastern patients had a higher proportion of proximal, differentiated and advanced gastric cancers. More total gastrectomies, larger extent of lymph node (LN) dissection, and higher number of retrieved LNs were found in the eastern patients. However, more Roux-en-Y anastomosis procedures during distal gastrectomy and intra-corporeal anastomosis were performed in the western patients. The west patients showed faster postoperative recovery than the eastern patients. The mortality rates of the western patients were comparable to those of the eastern patients. However, fewer III-IV complications were evident in the eastern centers. Multivariate analyses revealed that an elderly age, higher ASA score, and more blood loss were the significant independent risk factors of postoperative complications for eastern patients. However, for the western patients, the independent risk factors were neoadjuvant therapy, more retrieval LNs, and PT3-4 stage.

Conclusions: The selections and short-term surgical outcomes of LG for GC were widely different between East and West. To obtain more objective and accurate results, these differences should be considered in future international prospective studies.
\end{abstract}

Key words: stomach neoplasm; laparoscopic gastrectomy; surgical outcomes; postoperative complications; Clavien-Dindo classification

\section{Introduction}

Despite a global decline in incidence, there are about 2.2 million new cases of gastric cancer per year, resulting in 834 thousand deaths, making gastric cancer the second leading cause of cancer related mortality worldwide [1]. Since 1989, when Dubois et al. first reported their experience with laparoscopic 
cholecystectomy [2], abdominal surgery has increasingly moved toward a minimally invasive approach due to the many advantages regarding improvements in patient quality of life during the postoperative period. Laparoscopic approaches for gastric cancer in particular have been slowly accepted in the west, largely due to the lower incidence of gastric cancer in these countries. Currently, laparoscopic distal, subtotal, and total gastrecomy for early and advanced gastric cancer are emerging in the west with progressive acceptance among various groups [3-5]. Because several epidemiologic differences exist between east and west, the delayed acceptance of gastrectomy may be partly related to skepticism regarding the oncological efficacy of laparoscopic surgery. However, few studies have focused on the outcomes of the use of laparoscopic techniques for gastric cancer between Eastern and Western countries.

We present data from the International study group on Minimally Invasive surgery for GASTRIc Cancer (IMIGASTRIC) trial [6, 7], which is a multicenter study on gastric cancer developed to collect information on the surgical, clinical, and oncological features of patients undergoing gastrectomy with a robotic, laparoscopic, or open approach. To the best of our knowledge, this study is the first large-scale case-control study to compare the surgical outcomes of laparoscopic gastrectomy (LG) for gastric cancer between the east and west.

\section{Patients and Methods}

\section{Patients}

This study is a multi-institutional retrospective study that compares the short-term outcomes of LG for gastric carcinoma between eastern and western countries. Data collection started after reporting the specific study protocol. This study was registered at clinical trials.gov with a registration number of NCT02325453. This study was approved by the ethics Committee of CEAS Umbria. Tailored Web-based software was developed to standardize information, facilitate the process of data collection in a unified multi-institutional database, and guarantee the proper storage of patient's data. The purpose was to create an international registry with a high methodological quality. In the present study, the data regarding procedures performed up to the data collection (from January 2009 to August 2016) that entered into the IMIGASTRIC registry were analyzed. All involved centers were referral institutions with well-established gastric cancer programs and experience in minimally invasive surgery. All diagnostic and surgical interventions at these centers were performed according to international guidelines and the information stored in institutional prospective data collection systems. The inclusion criteria were as follows: gastric cancer reported in the biopsy report; upper endoscopy and CT scan for staging; either early or advanced gastric cancer; and curative surgery. The exclusion criteria were as follows: evidence of metastatic disease, a history of another surgery for gastric cancer, emergency surgery with bleeding or perforation, another malignancy, and surgery with palliative intent. Finally, 1858 eastern patients (from three centers) and 253 western patients (from ten centers) were included in the study. The surgical procedures were described in detail as follows: 1) total or subtotal gastrectomy was performed, according to the tumor location, size, and depth of invasion, and 2) D1+/D2 lymphadenectomy was undertaken according to the rules of the Japanese Research Society for Gastric Cancer $[8,9]$. Tumor staging was performed according to the 8th edition of the International Union against Cancer (UICC) TNM classification [10].

Patients were observed for 30 days following surgery, and short-term surgical outcomes including the operation time, estimated blood loss, postoperative complications, length of postoperative hospital stay, number of dissected lymph nodes (LNs) and clinicopathological characteristics were recorded in a web-based system (https://imigastric.logixsoftware.it/). Then, the data were extracted using pre-established IT tools. Postoperative complications were classified according to the revised version of the Clavien-Dindo classification system suggested by Dindo et al. [11, 12]. Types of postoperative complications were classified in accordance with the Japan Clinical Oncology Group Postoperative Complication Criteria according to the Clavien-Dindo classification ver. 2.0 [13]. The operation time was measured from the first skin incision to the closure of all skin incisions with skin staples. The mortality rate was defined as death within 30 days or in-hospital mortality when admitted beyond 30 days.

\section{Statistical analysis}

All statistical analyses were performed using SPSS v18.0 for Windows (SPSS Inc., Chicago, IL). The data are presented as the mean \pm standard deviation for continuous variables and as a number for categorical variables. For non-parametric data, variables were reported as the median (interquartile range). The differences in the patient demographics, surgical variables and pathological variables between the groups were calculated by using Fisher's exact test, the $t$-test, or the chi square test as appropriate. To evaluate the predictive factors for postoperative 
morbidity, a multivariate analysis was performed with binary logistic multiple regression analysis using dummy variables. $P$ values less than 0.05 were considered statistically significant.

Table 1. Patient demographics and tumor finding between the eastern and western centers

\begin{tabular}{|c|c|c|c|c|}
\hline \multirow[t]{2}{*}{ Characteristics } & \multicolumn{2}{|c|}{ All patients $(n=2111)$} & \multirow[t]{2}{*}{$\mathrm{X}^{2} / t$} & \multirow[t]{2}{*}{$\mathbf{P}$} \\
\hline & Eastern $(\mathrm{n}=1858)$ & Western $(n=253)$ & & \\
\hline Age & & & 154.04 & $<0.001$ \\
\hline$<40$ & $65(3.5)$ & $7(2.8)$ & & \\
\hline $40-75$ & $1607(86.5)$ & $149(58.9)$ & & \\
\hline$>75$ & 186(10.0) & 97(38.3) & & \\
\hline Mean $( \pm \mathrm{SD})$ & $61.0 \pm 11.2$ & $69.4 \pm 12.8$ & 10.92 & $<0.001$ \\
\hline Sex & & & 66.65 & $<0.001$ \\
\hline Male & 1407(75.7) & 130(51.4) & & \\
\hline Female & $451(24.3)$ & $123(48.6)$ & & \\
\hline Body mass index $(\mathrm{kg} / \mathrm{m} 2)$ & $22.2 \pm 3.2$ & $25.9 \pm 14.1$ & 7.24 & $<0.001$ \\
\hline ASA score & & & 1027.96 & 0.000 \\
\hline 1 & $1139(61.3)$ & $12(4.7)$ & & \\
\hline 2 & $646(34.8)$ & $57(22.5)$ & & \\
\hline 3 & $66(3.6)$ & 182(71.9) & & \\
\hline 4 & $7(0.4)$ & $2(0.8)$ & & \\
\hline Comorbidities & & & 3.48 & 0.062 \\
\hline Yes & $1276(68.7)$ & $159(62.8)$ & & \\
\hline No & $582(31.3)$ & $94(37.2)$ & & \\
\hline Previous surgery & & & 6.18 & 0.013 \\
\hline Yes & $413(22.2)$ & $74(29.2)$ & & \\
\hline No & $1445(77.8)$ & $179(70.8)$ & & \\
\hline Neoadjuvant chemotherapy & & & 66.60 & $<0.001$ \\
\hline Yes & $42(2.3)$ & $31(12.3)$ & & \\
\hline No & 1803(97.0) & $222(87.7)$ & & \\
\hline Tumor Location & & & 34.30 & $<0.001$ \\
\hline Upper & $593(31.9)$ & 39(15.4) & & \\
\hline Middle & $453(24.4)$ & $93(36.8)$ & & \\
\hline Lower & $812(43.7)$ & $121(47.8)$ & & \\
\hline Tumor size(cm) & $4.6 \pm 2.7$ & $4.8 \pm 5.7$ & 0.253 & 0.800 \\
\hline Histologic type & & & 16.73 & $<0.001$ \\
\hline Differentiated & 1308(70.4) & $146(57.7)$ & & \\
\hline Undifferentiated & $550(29.6)$ & $107(42.3)$ & & \\
\hline No. metastatic LNs & & & & \\
\hline pT stage & & & 137.91 & $<0.001$ \\
\hline $\mathrm{T} 1$ & $438(23.6)$ & $126(49.8)$ & & \\
\hline $\mathrm{T} 2$ & 200(10.8) & $35(13.8)$ & & \\
\hline T3 & $532(28.6)$ & 69(27.3) & & \\
\hline T4a & $685(36.9)$ & $18(7.1)$ & & \\
\hline $\mathrm{T} 4 \mathrm{~b}$ & $3(0.2)$ & $5(2.0)$ & & \\
\hline pN stage & & & 73.46 & $<0.001$ \\
\hline N0 & $655(35.3)$ & $156(61.7)$ & & \\
\hline N1 & $269(14.5)$ & $35(13.8)$ & & \\
\hline N2 & $307(16.5)$ & $27(10.7)$ & & \\
\hline N3a & $380(20.5)$ & 29(11.5) & & \\
\hline $\mathrm{N} 3 \mathrm{~b}$ & 247(13.3) & $6(2.4)$ & & \\
\hline pTNM stage & & & 110.72 & $<0.001$ \\
\hline IA & 365(19.6) & $110(43.5)$ & & \\
\hline IB & $152(8.2)$ & 33(13.0) & & \\
\hline IIA & $185(10.0)$ & 30(11.9) & & \\
\hline IIB & 206(11.1) & $24(9.5)$ & & \\
\hline IIIA & $189(10.2)$ & $16(6.3)$ & & \\
\hline IIIB & 299(16.1) & 28(11.1) & & \\
\hline IIIC & $462(24.9)$ & $12(4.7)$ & & \\
\hline
\end{tabular}

\section{Results}

\section{Patient clinicopathological characteristics}

The clinicopathological characteristics of the 2111 patients (1858 eastern vs. 253 western) are listed in Table 1. The western cohort comprised more elderly patients, more female patients, and patients with a higher body mass index (BMI) and a higher ASA score than the eastern cohort (all $\mathrm{P}<0.05)$. The proportion of patients with previous surgery and neoadjuvant chemotherapy was significantly higher in the western cohort than in the eastern cohort (both $\mathrm{P}<0.05)$. However, no significant differences in comorbidities were evident between the two cohorts $(\mathrm{P}>0.05)$.

There were more eastern patients than western patients had upper $1 / 3$ tumors (31.9\% in the east vs. $15.4 \%$ in the west; $\mathrm{P}<0.05)$. The eastern patients had a higher percentage of differentiated tumors with higher $\mathrm{T}$ stage and $\mathrm{N}$ stage compared to Western patients (all $\mathrm{P}<0.05)$. The median tumor size was similar between the cohorts $(\mathrm{P}>0.05)$.

\section{Surgical characteristics}

The type of resection, type of reconstruction and anastomosis approach were significantly different between the cohorts (all $\mathrm{P}<0.05$ ). The number of conversions to open surgeries, number of intraoperative blood transfusions, operation time, and volume of estimated blood loss were significantly lower in the eastern cohort than in the western cohort (all $\mathrm{P}<0.05)$. The number of patients who underwent D2 lymphadenectomy and R0 resection was significantly higher in the eastern cohort than in the western cohort (both $\mathrm{P}<0.05$ ). The number of received lymph nodes was higher in the east than in the west (33.5 \pm 13.1 vs. $21.2 \pm 12.6, \mathrm{P}<0.05)$ (Table 2$)$.

\section{Perioperative outcomes}

The proportion of postoperative transfusions was significantly lower in the east than in the west $(1.7 \%$ vs. $9.9 \%, \mathrm{P}<0.05)$. However, the time to NG tube removal, time to first flatus, time to start of a liquid diet, time to start of a soft diet, and the post-operative hospital stay were significantly shorter in the west than in the east (all $\mathrm{P}<0.001$, Table 2).

One or more complications occurred in 277 patients $(14.9 \%)$ in the east and 40 patients $(15.8 \%)$ in the west, and no statistical significance was evident $(\mathrm{P}>0.05)$. Anastomotic stenosis $(0.8 \%$ vs. $0.1 \%$, $\mathrm{p}=0.040)$ and urinary system complications $(2.0 \%$ vs. $0.4 \%, \mathrm{p}<0.001$ ) were more frequent in the west than in the east. However, no significant differences were evident for the other complications between the two cohorts $(\mathrm{P}>0.05)$.

According to the Clavien-Dindo classification, more postoperative major complications (grade III-IV) occurred in the west than in the east $(5.5 \%$ vs. $2.8 \%$, $\mathrm{P}<0.05$ ), but for grade I-II or grade $\mathrm{V}$ complications, no significant differences were evident between the two cohorts (both $\mathrm{P}>0.05$ ). Among the grade I-II 
complications, intra-abdominal bleeding $(\mathrm{P}=0.031)$, pleural effusion $(\mathrm{P}=0.002)$, and urinary system complications $(\mathrm{P}=0.003)$ occurred more frequently in the west than in the east, but the most frequent in the east were associated with pneumonia $(\mathrm{P}=0.010)$. Among the grade III-IV complications, more western patients than eastern patients suffered from anastomotic leakage $(2.4 \%$ vs. $0.5 \%, \mathrm{P}=0.002)$ and intestinal obstruction $(0.8 \%$ vs. $0.1 \%, \mathrm{P}=0.040)$ after LG, but no significant differences were evident for the other complications between the two cohorts $(\mathrm{P}>0.05)$ (Table 3).

Table 2. Surgical procedures between the eastern and western centers

\begin{tabular}{|c|c|c|c|c|}
\hline \multirow[t]{2}{*}{ Characteristics } & \multicolumn{2}{|c|}{ All patients $(n=2111)$} & \multirow[t]{2}{*}{$\mathrm{X}^{2} / t$} & \multirow[t]{2}{*}{$\mathbf{P}$} \\
\hline & $\begin{array}{l}\text { Eastern } \\
(\mathrm{n}=1858)\end{array}$ & $\begin{array}{l}\text { Western } \\
(\mathrm{n}=253)\end{array}$ & & \\
\hline Type of resection & & & 73.18 & $<0.001$ \\
\hline Distal gastrectomy & $811(43.6)$ & $170(67.2)$ & & \\
\hline Total gastrectomy & $1007(54.2)$ & $69(27.3)$ & & \\
\hline Proximal gastrectomy & $39(2.1)$ & $12(2.1)$ & & \\
\hline $\begin{array}{l}\text { Pylorus-preserving } \\
\text { gastrectomy }\end{array}$ & $1(0.1)$ & $2(0.8)$ & & \\
\hline Type of reconstruction & & & 1095.99 & $<0.001$ \\
\hline B-I & $682(36.7)$ & $0(0.0)$ & & \\
\hline B-II & $121(6.5)$ & $37(14.6)$ & & \\
\hline Rou-en-Y GJ & $8(0.4)$ & $133(52.6)$ & & \\
\hline Roux-en-Y EJ & $1007(54.2)$ & $66(26.1)$ & & \\
\hline Jejunal interposition & $0(0.0)$ & $4(1.6)$ & & \\
\hline Esophagogastrostomy & $39(2.1)$ & $11(4.3)$ & & \\
\hline Gastro-gastrostomy & $1(0.1)$ & $2(0.8)$ & & \\
\hline Anastomosis approach & & & 1019.24 & $<0.001$ \\
\hline intra-corporeal & $99(5.3)$ & $203(80.2)$ & & \\
\hline extra-corporeal & $1759(94.7)$ & $50(19.8)$ & & \\
\hline Extent of LN dissection & & & 603.87 & $<0.001$ \\
\hline D1 & $15(0.8)$ & $50(19.8)$ & & \\
\hline D1+ & $13(0.7)$ & $55(21.7)$ & & \\
\hline D2 & $1830(98.5)$ & $148(58.5)$ & & \\
\hline No. of retrieved lymph nodes & $33.5 \pm 13.1$ & $21.2 \pm 12.6$ & -14.06 & $<0.001$ \\
\hline Residual tumor & & & 12.54 & 0.002 \\
\hline $\mathrm{R} 1$ & $28(1.5)$ & $12(4.7)$ & & \\
\hline R0 & $1830(98.5)$ & $241(95.3)$ & & \\
\hline Conversion to open & & & 109.60 & $<0.001$ \\
\hline Yes & $1(0.1)$ & $16(6.3)$ & & \\
\hline No & 1857(99.9) & $237(93.7)$ & & \\
\hline Operation time (min) & $179.1 \pm 53.7$ & $217.0 \pm 84.6 .2$ & 9.71 & $<0.001$ \\
\hline Estimated blood loss (ml) & $74.8 \pm 101.0$ & $129.1 \pm 153.4$ & 6.99 & $<0.001$ \\
\hline Intraoperative blood transfusion & & & 22.43 & $<0.001$ \\
\hline Yes & $45(2.4)$ & $20(7.9)$ & & \\
\hline No & 1813(97.6) & $233(92.1)$ & & \\
\hline Postoperative transfusions & & & 56.425 & $<0.001$ \\
\hline Yes & $32(1.7)$ & $25(9.9)$ & & \\
\hline No & $1826(98.3)$ & $228(90.1)$ & & \\
\hline NG tube removal (days) & $5.1 \pm 3.0$ & $2.8 \pm 2.8$ & -4.31 & $<0.001$ \\
\hline Time to first flatus (days) & $3.7 \pm 1.3$ & $2.7 \pm 1.0$ & -6.95 & $<0.001$ \\
\hline Time to start of liquid diet(days) & $5.1 \pm 1.8$ & $2.3 \pm 2.1$ & -13.77 & $<0.001$ \\
\hline Time to start of soft diet (days) & $7.9 \pm 2.9$ & $3.3 \pm 1.9$ & -14.61 & $<0.001$ \\
\hline $\begin{array}{l}\text { Postoperative hospital stay } \\
\text { (days) }\end{array}$ & $13.1 \pm 8.1$ & $8.1 \pm 8.2$ & -9.93 & $<0.001$ \\
\hline
\end{tabular}

\section{Predictable risk factors for postoperative complications}

Univariate analysis and multivariate analysis were used to evaluate the factors that influenced postoperative complications. On univariate analysis, an age $>75$ years $(\mathrm{P}<0.001)$, a higher ASA score $(\mathrm{P}=0.002)$, more comorbidities $(\mathrm{P}=0.032)$, and an estimated blood loss $\geq 75 \mathrm{ml} \quad(\mathrm{P}=0.004)$ were significantly related to the occurrence of a postoperative complications in the east. However, in the west, comorbidities, previous surgery, neoadjuvant therapy, the anastomosis approach, combined resection, operation time, estimated blood loss, $\mathrm{pT}$ stage, and $\mathrm{pN}$ stage were significantly related to the occurrence of a postoperative complication (all $\mathrm{P}<0.05$ ) (Table 4).

Table 3. Postoperative complications between the eastern and western centers according to Clavien-Dindo classification

\begin{tabular}{|c|c|c|c|c|}
\hline \multirow[t]{2}{*}{ Characteristics } & \multicolumn{2}{|c|}{ All patients $(n=2111)$} & \multirow[t]{2}{*}{$\mathrm{X}^{2} / t$} & \multirow[t]{2}{*}{$\mathbf{P}$} \\
\hline & Eastern $(n=1858)$ & Western $(n=253)$ & & \\
\hline Total complications & $277(14.9)$ & $40(15.8)$ & 0.142 & 0.706 \\
\hline I-II complications & $223(12.0)$ & $24(9.5)$ & 1.264 & 0.243 \\
\hline Abdominal infection & $28(1.5)$ & $3(1.2)$ & 0.159 & 0.690 \\
\hline Anastomotic leakage & $21(1.1)$ & 0 & 2.888 & 0.089 \\
\hline Anastomotic site bleeding & $5(0.3)$ & $3(1.2)$ & 4.956 & 0.060 \\
\hline Anastomotic stenosis & 0 & $1(0.4)$ & 7.347 & 0.120 \\
\hline Delayed gastric emptying & $14(0.8)$ & $1(0.4)$ & 0.405 & 0.524 \\
\hline Intestinal obstruction & $22(1.2)$ & $1(0.4)$ & 1.286 & 0.257 \\
\hline Intra-abdominal bleeding & $2(0.1)$ & $2(1.3)$ & 10.247 & 0.031 \\
\hline Chylous leakage & $13(0.7)$ & 0 & 1.781 & 0.182 \\
\hline Pancreatic fistula & $4(0.2)$ & 0 & 0.546 & 1.000 \\
\hline Pleural effusion & 0 & $3(1.2)$ & 22.063 & 0.002 \\
\hline Pneumonia & $62(3.3)$ & $1(0.4)$ & 6.655 & 0.010 \\
\hline Wound infection & $25(1.3)$ & $2(0.8)$ & 0.461 & 0.543 \\
\hline Circulatory system & $4(0.2)$ & 0 & 0.546 & 1.000 \\
\hline Hematological system & $3(0.2)$ & $2(0.8)$ & 3.729 & 0.112 \\
\hline Urinary system & $8(0.4)$ & $5(2.0)$ & 8.693 & 0.003 \\
\hline Other & $12(0.6)$ & 0 & 1.643 & 0.200 \\
\hline III-IV complications & $52(2.8)$ & $14(5.5)$ & 5.499 & 0.019 \\
\hline Abdominal infection & $2(0.1)$ & $1(0.4)$ & 1.298 & 0.255 \\
\hline Anastomotic leakage & $10(0.5)$ & $6(2.4)$ & 9.950 & 0.002 \\
\hline Anastomotic site bleeding & $5(0.3)$ & $1(0.4)$ & 0.125 & 0.724 \\
\hline Anastomotic stenosis & $1(0.1)$ & $1(0.4)$ & 2.743 & 0.225 \\
\hline Intestinal obstruction & $1(0.1)$ & $2(0.8)$ & 8.516 & 0.040 \\
\hline Intra-abdominal bleeding & $10(0.5)$ & $1(0.4)$ & 0.088 & 0.767 \\
\hline Pneumonia & $12(0.6)$ & 0 & 1.643 & 0.200 \\
\hline Sepsis & $3(0.2)$ & $1(0.4)$ & 0.644 & 0.400 \\
\hline Wound infection & $2(0.1)$ & 0 & 0.273 & 1.000 \\
\hline Circulatory system & $4(0.2)$ & 0 & 0.546 & 1.000 \\
\hline Disseminated intravascular & $2(0.1)$ & 0 & 0.273 & 1.000 \\
\hline Acute renal failure & 0 & $1(0.4)$ & 7.347 & 0.120 \\
\hline V complications & $2(0.1)$ & $2(0.8)$ & 5.490 & 0.073 \\
\hline
\end{tabular}

Multivariate analysis found that an age $>75$ years $(\mathrm{P}<0.001)$, a higher ASA score $(\mathrm{P}=0.017)$, and an estimated blood loss $\geq 75 \mathrm{ml} \quad(\mathrm{P}=0.004)$ were independent risk factors for postoperative complications in the east. However, for the western patients, the independent risk factors were neoadjuvant therapy $(\mathrm{P}=0.012)$, the retrieval of more LNs $(\mathrm{P}=0.042)$, and pT3-4 stage $(\mathrm{P}<0.05)$ (Table 5).

\section{Discussion}

Currently, LG is becoming more widely used worldwide and some Eastern centers have become one of the standard surgical approaches for early gastric cancer [9]. LG for advanced gastric cancer has 
been developed successfully in many centers, and the number ongoing multicenter RCTs that focus on the safety and efficacy of LG in the east is increasing [14-16]. For the west, in 1992, Ohgami et al. reported the first laparoscopic wedge resection (LWR) for the treatment of early gastric cancer (EGC) [17].

Table 4. Univariate analysis of morbidity risk factors for eastern and western laparoscopy gastrectomy

\begin{tabular}{|c|c|c|c|c|c|c|}
\hline \multirow[t]{2}{*}{ Variable } & \multicolumn{3}{|c|}{ Eastern Centers } & \multicolumn{3}{|c|}{ Western Centers } \\
\hline & No & Yes & $\mathrm{P}$ & No & Yes & $\mathrm{P}$ \\
\hline Age & & & 0.000 & & & 0.370 \\
\hline$<40$ & $63(4.0)$ & $2(0.7)$ & & $7(3.3)$ & 0 & \\
\hline $40-75$ & $1381(87.3)$ & $226(81.6)$ & & $127(59.6)$ & $22(55.0)$ & \\
\hline$>75$ & $137(8.7)$ & $49(17.7)$ & & $79(37.1)$ & $18(45.0)$ & \\
\hline Sex & & & 0.120 & & & 0.878 \\
\hline Male & $1187(75.1)$ & $220(79.4)$ & & $109(51.2)$ & $21(52.5)$ & \\
\hline Female & $394(24.9)$ & $57(20.6)$ & & $104(48.8)$ & $19(47.5)$ & \\
\hline $\mathrm{BMI}(\mathrm{kg} / \mathrm{m} 2)$ & & & 0.636 & & & 0.198 \\
\hline$<25$ & $1275(80.6)$ & $220(79.4)$ & & $35(51.5)$ & $16(66.7)$ & \\
\hline$\geq 25$ & $306(19.4)$ & $57(20.6)$ & & $33(48.5)$ & $8(33.3)$ & \\
\hline ASA score & & & 0.002 & & & 0.764 \\
\hline 1 & $987(62.4)$ & $152(54.9)$ & & $11(5.2)$ & $1(2.5)$ & \\
\hline 2 & $541(34.2)$ & $105(37.9)$ & & $48(22.5)$ & $9(22.5)$ & \\
\hline $3-4$ & $53(3.4)$ & $20(7.2)$ & & $154(72.3)$ & $30(75.0)$ & \\
\hline Comorbidities & & & 0.032 & & & 0.004 \\
\hline No & $1101(69.6)$ & $175(63.2)$ & & $142(66.7)$ & $17(42.5)$ & \\
\hline Yes & $480(30.4)$ & $102(36.8)$ & & $71(33.3)$ & $23(57.5)$ & \\
\hline Previous surgery & & & 0.488 & & & 0.017 \\
\hline No & $1234(78.1)$ & $211(76.2)$ & & $157(73.7)$ & $22(55.0)$ & \\
\hline Yes & $347(21.9)$ & $66(23.8)$ & & $56(26.3)$ & $18(45.0)$ & \\
\hline Neoadjuvant therapy & & & 0.446 & & & 0.007 \\
\hline No & $1547(97.8)$ & $269(97.1)$ & & 192(90.1) & $30(75.0)$ & \\
\hline Yes & $34(2.2)$ & $8(2.9)$ & & $21(9.9)$ & $10(25.0)$ & \\
\hline Tumor location & & & 0.858 & & & 0.858 \\
\hline Upper & $504(31.9)$ & $89(32.1)$ & & $504(31.9)$ & $89(32.1)$ & \\
\hline Middle & $389(24.6)$ & $64(23.1)$ & & $389(24.6)$ & $64(23.1)$ & \\
\hline Total & $688(43.5)$ & $124(44.8)$ & & $688(43.5)$ & $124(44.8)$ & \\
\hline Tumor size (cm) & & & 0.890 & & & 0.205 \\
\hline$<4.6 \mathrm{~cm}$ & $829(52.4)$ & $144(52.0)$ & & $54(58.1)$ & $16(72.70)$ & \\
\hline$\geq 4.6 \mathrm{~cm}$ & $752(47.6)$ & $133(48.0)$ & & $39(41.9)$ & $6(27.3)$ & \\
\hline $\begin{array}{l}\text { Anastomosis } \\
\text { approach }\end{array}$ & & & 0.516 & & & 0.027 \\
\hline Intra-corporeal & $82(5.2)$ & $17(6.1)$ & & $176(82.6)$ & $27(67.5)$ & \\
\hline Extra-corporeal & $1499(94.8)$ & $260(93.9)$ & & $37(17.4)$ & $13(32.5)$ & \\
\hline Type of resection & & & 0.932 & & & 0.798 \\
\hline Distal gastrectomy & $687(43.5)$ & $124(44.8)$ & & $145(68.1)$ & $25(62.5)$ & \\
\hline Total gastrectomy & $859(54.3)$ & $148(53.4)$ & & $56(26.3)$ & $13(32.5)$ & \\
\hline $\begin{array}{l}\text { Proximal } \\
\text { gastrectomy }\end{array}$ & $34(2.2)$ & $5(1.8)$ & & $10(4.7)$ & $2(5.0)$ & \\
\hline $\begin{array}{l}\text { Pylorus-preserving } \\
\text { gastrectomy }\end{array}$ & $1(0.1)$ & 0 & & $2(0.19$ & 0 & \\
\hline $\begin{array}{l}\text { Type of } \\
\text { reconstruction }\end{array}$ & & & 0.923 & & & 0.630 \\
\hline B-I & $581(36.7)$ & $101(36.5)$ & & 0 & 0 & \\
\hline B-II & $99(6.3)$ & $22(7.9)$ & & $34(16.0)$ & $3(7.5)$ & \\
\hline Roux-en-Y GJ & $7(0.4)$ & $1(0.4)$ & & $111(52.1)$ & $22(55.0)$ & \\
\hline Roux-en-Y EI & $869(54.3)$ & $148(53.4)$ & & $56(26.3)$ & $14(35.0)$ & \\
\hline & $34(2.2)$ & $5(1.8)$ & & $10(4.7)$ & $1(2.5)$ & \\
\hline Esophagogastrostomy & & & & & & \\
\hline Gastrogastrostomy & $1(0.1)$ & 0 & & $2(0.9)$ & 0 & \\
\hline $\begin{array}{l}\text { Type of } \\
\text { lymphadenectomy }\end{array}$ & & & 0.659 & & & 0.889 \\
\hline $\mathrm{D} 1 / \mathrm{D} 1+$ & $23(1.5)$ & $5(1.8)$ & & $88(41.3)$ & $17(42.5)$ & \\
\hline $\mathrm{D} 2$ & $1558(98.5)$ & $272(98.2)$ & & $125(58.7)$ & $23(57.5)$ & \\
\hline Combined resection & & & 0.058 & & & 0.006 \\
\hline No & $1419(89.8)$ & $238(85.9)$ & & $178(83.6)$ & $26(65.0)$ & \\
\hline Yes & $162(10.2)$ & $39(14.1)$ & & $35(16.4)$ & $14(35.0)$ & \\
\hline Operation time (min) & & & 0.151 & & & 0.028 \\
\hline$<180$ & $1167(73.8)$ & 193(69.7) & & $120(56.3)$ & $15(37.5)$ & \\
\hline$\geq 180$ & $414(26.2)$ & $84(30.3)$ & & $93(43.7)$ & $25(62.5)$ & \\
\hline
\end{tabular}

\begin{tabular}{|c|c|c|c|c|c|c|c|}
\hline \multirow[t]{2}{*}{ Variable } & \multicolumn{4}{|c|}{ Eastern Centers } & \multicolumn{3}{|c|}{ Western Centers } \\
\hline & No & Yes & $\mathrm{P}$ & & No & Yes & $\mathrm{P}$ \\
\hline $\begin{array}{l}\text { Estimated blood loss } \\
\text { (ml) }\end{array}$ & & & 0.004 & & & & 0.006 \\
\hline$<75$ & $1277(80.8)$ & 203(73.3) & & & $121(72.0)$ & $18(48.6)$ & \\
\hline$\geq 75$ & $304(19.2)$ & $74(2.7)$ & & & $47(28.0)$ & $19(51.4)$ & \\
\hline No. of retrieved LNs & & & 0.102 & & & & 0.086 \\
\hline$<34$ & $881(55.7)$ & $169(61.0)$ & & & $127(59.6)$ & $18(45.0)$ & \\
\hline$\geq 34$ & $700(44.3)$ & $108(39.0)$ & & & $86(40.4)$ & $22(55.0)$ & \\
\hline Histological & & & 0.775 & & & & 0.775 \\
\hline Differentiated & $1115(70.5)$ & 193(69.7) & & & $1115(70.5)$ & $193(69.7)$ & \\
\hline Undifferentiated & $466(29.5)$ & $84(30.3)$ & & & $466(29.5)$ & $84(30.3)$ & \\
\hline pT stage & & & 0.672 & & & & 0.046 \\
\hline $\mathrm{T} 1$ & $373(23.6)$ & $65(23.5)$ & & & $106(49.8)$ & $20(50.0)$ & \\
\hline $\mathrm{T} 2$ & 176(11.1) & $24(8.7)$ & & & $32(15.0)$ & $3(7.5)$ & \\
\hline $\mathrm{T} 3$ & $447(28.3)$ & $85(30.7)$ & & & $60(28.2)$ & $9(22.5)$ & \\
\hline $\mathrm{T} 4$ & $585(40.0)$ & $103(37.2)$ & & & $15(7.0)$ & $8(20.0)$ & \\
\hline $\mathrm{pN}$ stage & & & 0.140 & & & & 0.030 \\
\hline No & $570(36.1)$ & $85(30.7)$ & & & $132(62.0)$ & $24(60.0)$ & \\
\hline N1 & $224(14.2)$ & $45(16.2)$ & & & $33(15.5)$ & $2(5.0)$ & \\
\hline $\mathrm{N} 2$ & $266(16.8)$ & $41(14.8)$ & & & $24(11.3)$ & $3(7.5)$ & \\
\hline N3a & $310(19.6)$ & $70(25.3)$ & & & $19(8.9)$ & $10(25.0)$ & \\
\hline N3b & 211(13.3) & $36(13.0)$ & & & $5(2.3)$ & $1(2.5)$ & \\
\hline pTNM stage & & & 0.683 & & & & 0.188 \\
\hline IA & $311(19.7)$ & $54(19.5)$ & & I & $122(57.3)$ & $21(52.5)$ & \\
\hline IB & $135(8.5)$ & $17(6.1)$ & & II & $48(22.5)$ & $6(15.0)$ & \\
\hline IIA & $162(10.2)$ & $23(8.3)$ & & III & $43(20.2)$ & $13(32.5)$ & \\
\hline IIB & $170(10.8)$ & $36(13.0)$ & & & & & \\
\hline IIIA & $160(10.1)$ & $29(10.5)$ & & & & & \\
\hline IIIB & $254(16.1)$ & $45(16.2)$ & & & & & \\
\hline IIIC & $389(24.6)$ & $73(26.4)$ & & & & & \\
\hline
\end{tabular}

Table 5. Multivariate analysis of the morbidity for eastern and western laparoscopy gastrectomy

\begin{tabular}{lllllll}
\hline Variable & $\mathrm{B}$ & $\mathrm{S} . \mathrm{E}$ & Wals & $\mathrm{P}$ & $\mathrm{OR}$ & $95 \% \mathrm{CI}$ \\
\hline Eastern centers & & & & & & \\
Age & 0.773 & 0.173 & 20.064 & 0.000 & 2.166 & $1.545-3.038$ \\
ASA score & 0.369 & 0.155 & 5.660 & 0.017 & 1.446 & $1.067-1.960$ \\
Comorbidities & 0.198 & 0.203 & 0.953 & 0.329 & 1.219 & $0.819-1.814$ \\
Combined resection & -0.343 & 0.195 & 3.092 & 0.079 & 0.710 & $0.484-1.040$ \\
Estimated blood loss & 0.439 & 0.152 & 8.353 & 0.004 & 1.551 & $1.152-2.089$ \\
$\begin{array}{l}\text { Constant } \\
\text { Western centers }\end{array}$ & -3.842 & 0.532 & 52.222 & 0.000 & 0.021 & \\
Comorbidities & -0.244 & 0.592 & 0.169 & 0.681 & 0.784 & $0.245-2.502$ \\
$\begin{array}{l}\text { Previous surgery } \\
\text { Neoadjuvant }\end{array}$ & -0.617 & 0.686 & 0.810 & 0.368 & 0.539 & $0.141-2.069$ \\
therapy & -1.471 & 0.584 & 6.345 & 0.012 & 0.230 & $0.073-0.722$ \\
$\begin{array}{l}\text { Anastomosis } \\
\text { approach }\end{array}$ & -0.583 & 0.540 & 1.167 & 0.280 & 0.558 & $0.194-1.608$ \\
Combined resection & -0.179 & 0.583 & 0.094 & 0.759 & 0.836 & $0.267-2.623$ \\
Operation time & -0.142 & 0.685 & 0.043 & 0.836 & 0.867 & $0.227-3.322$ \\
Estimated blood loss & -0.677 & 0.650 & 1.086 & 0.297 & 0.508 & $0.142-1.816$ \\
No. of retrieved LN & -0.933 & 0.459 & 4.136 & 0.042 & 0.394 & $0.160-0.967$ \\
pT stage & & & 7.792 & 0.051 & & \\
pT1 vs pT2 & -0.866 & 0.781 & 1.227 & 0.268 & 0.421 & $0.091-1.946$ \\
pT1 vs pT3 & -1.926 & 0.981 & 3.855 & 0.050 & 0.146 & $0.021-0.997$ \\
pT1 vs pT4 & -1.931 & 0.796 & 5.889 & 0.015 & 0.145 & $0.030-0.690$ \\
pN stage & & & 5.827 & 0.212 & & \\
pN0 vs pN1 & 0.102 & 1.736 & 0.003 & 0.953 & 1.107 & $0.037-33.265$ \\
pN0 vs pN2 & -1.544 & 1.861 & 0.688 & 0.407 & 0.214 & $0.006-8.204$ \\
pN0 vs pN3a & -0.987 & 1.817 & 0.295 & 0.587 & 0.373 & $0.011-13.116$ \\
pN0 vs pN3b & 0.470 & 1.717 & 0.295 & 0.784 & 1.600 & $0.055-46.320$ \\
Constant & 3.019 & 1.791 & 2.843 & 0.092 & 20.481 & \\
\hline
\end{tabular}

Recently, LG has become an important surgical procedure for radical gastrectomy in many western centers. However, no reports on the efficacy of LAG between the east and west centers are available. This study is the first large case-control study using multicenter clinical research data (IMIGASTRIC trial), and can be used as a preliminary basis for future 
prospective studies. In this study, the 1858 patients in the east and 253 patients in the west had significant differences in demographic, clinical, and pathologic characteristics. Patient characteristics such as age, and BMI and the ASA score were higher in the west. This result may be due to epidemiological differences between the east and west [18]. However, differences in the selection of laparoscopic surgery cases between the east and west may have influenced the results. Hamashima C KY et al. found that the age of population-based screening is early in eastern countries, resulting in an age of onset that is lower in the east than in the west [19]. The higher BMI in the western patient group may correspond to an overall higher BMI in the western population. Regarding the pathological features, more undifferentiated earlier-stage tumors were evident in the west than in the east for laparoscopic surgery, which was consistent with the study by Strong VE et al. [20]. In addition, we found that the majority of patients in both cohorts had tumors in the distal stomach, but the proportion of upper $1 / 3$ tumors was significantly higher in the east than in the west $(31.9 \%$ vs. $15.4 \%$, $\mathrm{P}<0.05)$, which was contrary to previous studies [18]. Laparoscopic total gastrectomy for proximal gastric cancer is a difficult procedure, and fewer western centers may choose this approach for laparoscopic surgery. However, in the east, the number of gastric cancer cases was higher, the patients were younger and had less BMI, and these factors increased the number of options for laparoscopic surgery. Recently, several studies have shown that the incidence of proximal gastric cancer in the east has increased annually [21-23], possibly explaining higher proportion Eastern patients undergoing proximal and total gastrectomy in the current study.

The number of conversion to open surgeries, number of blood transfusions, and volume of estimated blood loss were higher in the west than in the east, and the operation time was longer in the west than in the east; however, the number of LN dissections was lower in the west than in the east. These observations may be attributed to differences in the patient characteristics between the east and west. More options for the treatment of early gastric cancer exist in the west than in the east, so the extent of LN dissection is smaller. Due to several factors in the west, such as the lower overall incidence of gastric cancer and higher BMI, which can increase the difficulty of laparoscopic operation, the number of conversions to open surgery, the operation time, and the volume of estimated blood loss were higher in the west than in the east. In the east, the data have suggested a learning curve number of 20-40 procedures [24]. In another report from the west,
Kunisaki $C$ et al. showed that the learning curve for surgeons comprised approximately 60 cases, after which the operation time significantly improved with equivalent LN retrievals and surgical outcomes [25]. The incidence of gastric cancer is lower in the west than in the east. However, the learning curve for performing minimally invasive gastrectomy requires more cases. When we compared postoperative recovery between the west and east, we found that the time to NG tube removal, time to first flatus, time to start of a liquid diet, time to the start of a soft diet and postoperative hospital stay were significantly shorter in the west than in the east, which may be related to the early implementation and application of enhanced recovery protocols (ERAS).

The incidence of postoperative complications is still the most frequently used surrogate marker of the 'quality' of surgery. Previous reported morbidity rates for laparoscopic surgery vary from 6.1 to $25.4 \%$ [15, 26-28]. In this study, the postoperative morbidity was $14.9 \%$ in the east and $15.8 \%$ in the west, without significant differences. When the complications were categorized with the Clavien-Dindo classification, we found that grade III-IV complications occurred more frequently in the west than in the east $(5.5 \%$ vs. $2.8 \%$, $\mathrm{P}<0.05)$, which mainly contributed to the higher incidence of anastomotic leakage and intestinal obstruction. In the west, due to early drain removal or no drain placed after surgery, when anastomotic leakage or other complications occur, more invasive procedures, such as abdominal paracentesis and drainage were performed, and lead to increases in the grade of complications. Multivariate analysis further revealed that an elderly age, a higher ASA score, and a higher estimated blood loss were the independent risk factors for postoperative complications in the east, which was consistent with several previous eastern studies $[29,30]$. In the west, neoadjuvant therapy, pT3-4 and the number of LN dissections were the independent risk factors for postoperative morbidity, which suggested that advanced gastric cancer and a wider range of LN dissection would increase the incidence of postoperative complications in the western centers. Haskins and Badgwell et al. reported that postoperative morbidity and mortality were not increased by neoadjuvant therapy [31, 32]. However, the open and laparoscopic groups were not analyzed separately in these studies, which may have caused the results to be different from those of the present study. Most of the gastric cancer patients who underwent neoadjuvant therapy had more advanced stages and needed larger extents of LN dissection, increasing the difficulty of the operation and the likelihood of morbidity, especially for laparoscopic surgery with higher technical requirements. 
In conclusion, distinct differences are evident between the east and west regarding the clinicopathological characteristics and short-outcomes of laparoscopic surgery for gastric cancer. Although no significant differences in overall complications were evident between the two cohorts, the major postoperative complications (grade III-IV) and risk factors for morbidity were significantly different. Therefore, for the prospective study of the efficacy of laparoscopic radical gastrectomy for gastric cancer between the east and west, we should recognize the objective factors and pay more attention to the surgical indications and case selections to obtain more objective and accurate results.

\section{Acknowledgements}

We thank the all the centers which contributed to the IMIGASTRIC trial. And the follow-up office established by the Department of gastric surgery, Fujian Medical University Union Hospital, Fuzhou, Fujian Province, China.

\section{Ethics approval and consent to participate}

The study was approved by the ethics Committee of CEAS Umbria. Written consent was obtained from all patients, whose information was stored in the hospital database and used for our research.

\section{Consent for publication}

Written consent was given by the patients and their relatives to use their information in a research study and publish it.

\section{Availability of data and material}

The data that support the findings of this study are available from the corresponding author upon reasonable request.

\section{Funding}

This work was supported by Scientific and technological innovation joint capital projects of Fujian Province, China (No.2016Y9031). The youth research project of Fujian Provincial Health and Family Planning Commission (2014-1-48). CARIT Foundation (Fondazione Cassa di Risparmio di Terni e Nami), No. 0024137.

\section{Author contributions}

Lin JX, Lin JP, Desiderio J, Li P, Parisi A, and Huang CM conceived of the study, analyzed the data, and drafted the manuscript; Xie JW, Gemini A, Wang $\mathrm{JB}$, and Zheng $\mathrm{CH}$ helped revise the manuscript critically for important intellectual content; Lu J, Chen QY, Cao LL, Lin M and Tu RH helped collect data and design the study.

\section{Competing Interests}

The authors have declared that no competing interest exists.

\section{References}

1. GBD 2016 Disease and Injury Incidence and Prevalence Collaborators. Global, regional, and national incidence, prevalence, and years lived with disability for 328 diseases and injuries for 195 countries, 1990-2016: a systematic analysis for the Global Burden of Disease Study 2016. Lancet. 2017; 390(10100):1211-1259.

2. Hsieh CH. Laparoscopic Cholecystectomy for Patients with Chronic Obstructive Pulmonary Disease. J Laparoendosc Adv Surg Tech A. 2003; 1: 5-9.

3. Kawamura H, Homma S, Yokota R et al. Inspection of Safety and Accuracy of D2 Lymph Node Dissection in Laparoscopy-Assisted Distal Gastrectomy. World J Surg. 2008; 11: 2366-70.

4. Tanimura S, Higashino M, Fukunaga $Y$ et al. Laparoscopic Gastrectomy for Gastric Cancer: Experience with More than 600 Cases. Surg Endosc. 2008; 5: 1161-4.

5. Orditura M, Galizia G, Sforza V et al. Treatment of Gastric Cancer. World J Gastroenterol. 2014; 7: 1635-49.

6. Parisi A, Reim D, Borghi F et al. Minimally Invasive Surgery for Gastric Cancer: A Comparison Between Robotic, Laparoscopic and Open Surgery. World J Gastroenterol. 2017; 13: 2376-84.

7. Desiderio J, Jiang ZW, Nguyen NT et al. Robotic, Laparoscopic and Open Surgery for Gastric Cancer Compared On Surgical, Clinical and Oncological Outcomes: A Multi-Institutional Chart Review. A Study Protocol of the International Study Group On Minimally Invasive Surgery for GASTRIc Cancer-IMIGASTRIC. Bmj Open. 2015; 10: e8198.

8. Japanese GCA. Japanese Classification of Gastric Carcinoma - 2Nd English Edition -. Gastric Cancer. 1998; 1: 10-24.

9. Japanese GCA. Japanese Classification of Gastric Carcinoma: 3Rd English Edition. Gastric Cancer. 2011; 2: 101-12.

10. Amin MB ES. AJCC Cancer Staging Manual. 8Th Ed, New York: Springer, 2016.

11. Dindo D, Demartines N, Clavien PA. Classification of Surgical Complications: A New Proposal with Evaluation in a Cohort of 6336 Patients and Results of a Survey. Ann Surg. 2004; 2: 205-13.

12. Clavien PA, Sanabria JR, Strasberg SM. Proposed Classification of Complications of Surgery with Examples of Utility in Cholecystectomy. Surgery. 1992; 5: 518-26.

13. [Internet] Japan Clinical Oncology Group. Postoperative Complication Criteria according to Clavien-Dindo Classification ver. 2.0. http://www.jcog.jp/doctor/tool/Clavien_Dindo.html.

14. Kim YW, Baik YH, Yun YH et al. Improved Quality of Life Outcomes After Laparoscopy-Assisted Distal Gastrectomy for Early Gastric Cancer: Results of a Prospective Randomized Clinical Trial. Ann Surg. 2008; 5: 721-7.

15. Kim HH, Hyung WJ, Cho GS et al. Morbidity and Mortality of Laparoscopic Gastrectomy Versus Open Gastrectomy for Gastric Cancer: An Interim Report--A Phase III Multicenter, Prospective, Randomized Trial (KLASS Trial). Ann Surg. 2010; 3: 417-20.

16. Hu Y, Huang C, Sun Y et al. Morbidity and Mortality of Laparoscopic Versus Open D2 Distal Gastrectomy for Advanced Gastric Cancer: A Randomized Controlled Trial. J Clin Oncol. 2016; 12: 1350-7.

17. Ohgami M, Otani Y, Kumai K, Kubota T, Kim YI, Kitajima M. Curative Laparoscopic Surgery for Early Gastric Cancer: Five Years Experience. World J Surg. 1999; 2: 187-92, 192-3.

18. Ichikura T, Ogawa T, Kawabata $T$, Chochi K, Sugasawa H, Mochizuki H. Is Adenocarcinoma of the Gastric Cardia a Distinct Entity Independent of Subcardial Carcinoma? World J Surg. 2003; 3: 334-8.

19. Hamashima C, Kim Y, Choi KS. Comparison of guidelines and management for gastric cancer screening between Korea and Japan. ISPOR 20th Annual International Meeting. Philadelphia; 2015.

20. Strong VE, Wu AW, Selby LV et al. Differences in Gastric Cancer Survival Between the U.S. And China. J Surg Oncol. 2015; 1: 31-7.

21. Abnet CC, Freedman ND, Hu N et al. A Shared Susceptibility Locus in PLCE1 at 10Q23 for Gastric Adenocarcinoma and Esophageal Squamous Cell Carcinoma. Nat Genet. 2010; 9: 764-7.

22. Wang LD, Zhou FY, Li XM et al. Genome-Wide Association Study of Esophageal Squamous Cell Carcinoma in Chinese Subjects Identifies Susceptibility Loci at PLCE1 and C20orf54. Nat Genet. 2010; 9: 759-63.

23. Qiao YL, Dawsey SM, Kamangar F et al. Total and Cancer Mortality After Supplementation with Vitamins and Minerals: Follow-Up of the Linxian General Population Nutrition Intervention Trial. J Natl Cancer Inst. 2009; 7: 507-18.

24. Kim HG, Park JH, Jeong SH et al. Totally Laparoscopic Distal Gastrectomy After Learning Curve Completion: Comparison with Laparoscopy-Assisted Distal Gastrectomy. J Gastric Cancer. 2013; 1: 26-33.

25. Kunisaki C, Makino $\mathrm{H}$, Yamamoto $\mathrm{N}$ et al. Learning Curve for Laparoscopy-Assisted Distal Gastrectomy with Regional Lymph Node Dissection for Early Gastric Cancer. Surg Laparosc Endosc Percutan Tech. 2008; 3: 236-41. 
26. Kitano S, Shiraishi N, Uyama I, Sugihara K, Tanigawa N. A Multicenter Study On Oncologic Outcome of Laparoscopic Gastrectomy for Early Cancer in Japan. Ann Surg. 2007; 1: 68-72.

27. Miura S, Kodera $Y$, Fujiwara $M$ et al. Laparoscopy-Assisted Distal Gastrectomy with Systemic Lymph Node Dissection: A Critical Reappraisal From the Viewpoint of Lymph Node Retrieval. J Am Coll Surg. 2004; 6: 933-8.

28. Sugimoto $\mathrm{M}$, Kinoshita $\mathrm{T}$, Shibasaki $\mathrm{H}$ et al. Short-Term Outcome of Total Laparoscopic Distal Gastrectomy for Overweight and Obese Patients with Gastric Cancer. Surg Endosc. 2013; 11: 4291-6.

29. Jeong O, Ryu SY, Choi WY, Piao Z, Park YK. Risk Factors and Learning Curve Associated with Postoperative Morbidity of Laparoscopic Total Gastrectomy for Gastric Carcinoma. Ann Surg Oncol. 2014; 9: 2994-3001.

30. Lee KG, Lee HJ, Yang JY et al. Risk Factors Associated with Complication Following Gastrectomy for Gastric Cancer: Retrospective Analysis of Prospectively Collected Data Based On the Clavien-Dindo System. J Gastrointest Surg. 2014; 7: 1269-77.

31. Badgwell B, Ajani J, Blum $\mathrm{M}$ et al. Postoperative Morbidity and Mortality Rates are Not Increased for Patients with Gastric and Gastroesophageal Cancer Who Undergo Preoperative Chemoradiation Therapy. Ann Surg Oncol. 2016; 1: 156-62.

32. Haskins IN, Kroh MD, Amdur RL, Ponksy JL, Rodriguez JH, Vaziri K. The Effect of Neoadjuvant Chemoradiation on Anastomotic Leak and Additional 30-Day Morbidity and Mortality in Patients Undergoing Total Gastrectomy for Gastric Cancer. J Gastrointest Surg. 2017. 